\title{
What we learn in time of pestilence
}

\section{Rachel Ellaway ${ }^{1} \cdot$ Jennifer Cleland $^{2} \cdot$ Martin Tolsgaard $^{3}$}

Published online: 29 April 2020

(c) Springer Nature B.V. 2020

We take the title for this editorial from the English translation of Albert Camus' 1947 novel La Peste (Camus 1972). We were originally going to report on the Journal's Fast Track review approach in this editorial. However, events have overtaken us, and we will instead, perhaps predictably, reflect on the current pandemic in the context of health professions education. Clearly the 2020 COVID-19 pandemic has been deeply disruptive to Health Professional Education (HPE) around the world. Classes have been cancelled or moved online, exams have been postponed, and many learners and teachers have volunteered or been drafted to help in the current emergency.

Articles on how educators have responded or might respond to COVID-19 are already appearing in the literature along with a growing body of commentaries and letters describing how everything either has changed or how everything is going to change as a result of COVID-19. Many journals have set up quick response formats so that they can disseminate new and emerging practices and solutions quickly and efficiently. At Advances we have not and will not be doing this. The 'why not?' bears some explanation.

It is always a concern that the urgent displaces the important (with apologies to Eisenhower). A key concern in the current situation is how our understanding of both the virus and its systemic impacts have changed and continue to change. Already there has been a rush to publish and make definitive statements about how COVID-19 has impacted HPE. However, rather than rushing out reports of how individual institutions have adapted to "keep the [educational] show on the road", we believe that the depth and breadth of the impact and implications of COVID-19 in relation to HPE need a more measured and thoughtful response, not least because it may be some time before we can fully encompass its true scope and impact. Moreover, we can be sure that pre-COVID problems and concerns about many different aspects of HPE teaching, learning and assessment have not gone away. The current situation may cast them in a new light in the immediate and possibly even the longer term, but it is very unlikely to resolve or eradicate them.

Given the remit of the Journal, we will leave the 'how to ...' or 'how we ...' papers to other more practically focused journals. We would encourage those authors seeking to explore these issues through this Journal to do so in ways that reflect the deep

Rachel Ellaway

rachel.ellaway@ucalgary.ca

1 Cumming School of Medicine, University of Calgary, Calgary, AB, Canada

2 Lee Kong Chian School of Medicine, Nanyang Technological University, Singapore, Singapore

3 Centre for Clinical Education, University of Copenhagen, Copenhagen, Denmark 
theory-informed thinking that we see as our hallmark. For instance, theory could play a critical role in developing our understanding of how and at what cost organizations and the individuals within them have changed and adapted to COVID-19; if, and if so, how educational philosophies or ideologies have shifted; how we think about individual and collective resilience and wellness; how professional identity formation may have been reshaped during this period of disruption; and indeed, how we think about the purposes and principles of health professional education. Theory informs methods and we would look to our authors to select and/or develop methodologies and methods that allow the multitude of questions emerging from COVID-19 events to be explored carefully and compellingly.

To that end, we seek papers that do not simply describe, but explain, model, and predict, and that deepen our understanding of HPE, both during the pandemic in its many phases and in the times that follow. While there is likely to be much valuable work about the impacts of the pandemic on training, on the adaptation and adaptability of programs and participants, and the impacts and implications of those changes, there are many more areas worthy of exploration. For instance, the critical importance of public health and its use of data and modeling has been a notable aspect of our responses to COVID-19. What are the implications of this in HPE? There are many indirect issues worthy of exploration such as the likely economic pressures that a significant world economic downturn is likely to have on education, whether the emergency changes made will persist (such as a much more extensive use of online educational tools and environments), and how we might reconsider how we model and teach professionalism and ethics when many healthcare providers have lost their lives and many more are working under very difficult circumstances. Indeed, we might be well to reconsider the most fundamental issues in HPE as to how we prepare for and recognize when a learner is ready to practice.

Authors might also ask how we prepare physicians who are well into their careers to transition to different types of working, potentially in roles and in environments which they may have little or no recent experience, and they may also ask at what cost these changes are made. Authors may also explore how the different adaptions to medical education practices have impacted learners' core skills development or their professional identity formation. At a wider systems level, authors might explore how curriculum leaders decided which educational activities were to be added, dropped or changed and the drivers, costs, and impacts of those decisions. It may be that some will present findings that show that HPE was found wanting and needs to undergo fundamental change, while others may argue that HPE was well prepared, and may even have been strengthened by the current crisis. Finally, we would be interested in studies exploring how the pandemic has affected HPE research, its activities, funding opportunities, and its place in HPE as a whole.

There has clearly been a terrible human toll from the pandemic in terms of death, the suffering of patients and their loved ones, and the healthcare workers who cared for them, all of which contribute to unprecedented psychological, social, and economic impacts. And yet, as Churchill once said, "never let a good crisis go to waste". There is a window of opportunity afforded by the pandemic and our responses to it to examine much of what we do in HPE in a new light and from perspectives that have not previously been available to us. COVID-19 may provide an opportunity for HPE to demonstrate its worth to society more than ever. However, COVID-19 is not all the world, our many other problems and challenges persist, and they need robust scholarly responses too. Now, as much as we are tired and afraid as individuals and as a species, we need to respond with all the power and insight our sciences can bring to bear. Let that be our challenge to the community; to honour the suffering but at the same time advance our thinking and practice as best we can. Only by doing so will the current situation and its ramifications help us rethink how 
we educate, and question what it is we need to teach, and what it is we are preparing our learners for. The full quote from Camus is: "To state quite simply what we learn in time of pestilence: that there are more things to admire in men than to despise."

\section{AHSE 25th special anniversary issue}

Before we close, we would also like to invite submissions to our 25th Special Anniversary issue that is intended to provide an opportunity to reflect on the ways in which Advances in Health Sciences Education has contributed to the field over the years. We invite submissions that speak to the role of disciplinary and interdisciplinary perspectives in the study of health professions education, in particular those that reflect our focus on theory and method from diverse social and behavioural science disciplines. Papers could take a variety of formats: a review of the history of contributions from disciplines such as psychology and sociology, introduction of new disciplinary/or interdisciplinary thinking, or original studies exemplifying the use of theory and/or methods from a discipline. We would also be interested in contributions to HPE from fields such as economics, business, engineering, big data, and artificial intelligence among others. Further details on the Journal website.

\section{Reference}

Camus, A. (1972). The Plague (La Peste) (S. Gilbert, Trans.) New York: NY. Vintage Books.

Publisher's Note Springer Nature remains neutral with regard to jurisdictional claims in published maps and institutional affiliations. 